\title{
MATLIS DUALS OF TOP LOCAL COHOMOLOGY MODULES
}

\author{
MICHAEL HELLUS AND JÜRGEN STÜCKRAD
}

(Communicated by Bernd Ulrich)

\begin{abstract}
In the first section of this paper we present generalizations of known results on the set of associated primes of Matlis duals of local cohomology modules; we prove these generalizations by using a new technique. In section 2 we compute the set of associated primes of the Matlis dual of $\mathrm{H}_{J}^{d-1}(R)$, where $R$ is a $d$-dimensional local ring and $J \subseteq R$ an ideal such that $\operatorname{dim}(R / J)=1$ and $\mathrm{H}_{J}^{d}(R)=0$.
\end{abstract}

\section{INTRODUCTION AND MOTIVATION}

In algebraic geometry one investigates the number of equations needed to cut out a given variety; this number is called the arithmetic rank ara; thus for a given ideal $I$ in a noetherian ring $R$ we have

$$
\operatorname{ara}(I)=\min \left\{h \in \mathbb{N} \mid \exists r_{1}, \ldots, r_{h} \in I: \sqrt{I}=\sqrt{\left(r_{1}, \ldots, r_{h}\right) R}\right\} .
$$

One always has $\operatorname{ara}(I) \geq \operatorname{codim}(I)$. We say $I$ (or the variety given by $I$ ) is a (settheoretic) complete intersection if $\operatorname{ara}(I)=\operatorname{codim}(I)$ holds. For an $R$-module $M$, we denote by $\mathrm{H}_{I}^{i}(M)$ the $i$-th local cohomology module of $M$ supported in $I$. Let $n$ be a natural number; then $\operatorname{ara}(I) \leq n$ implies $0=\mathrm{H}_{I}^{n+1}(R)=\mathrm{H}_{I}^{n+2}(R)=\ldots$, but the converse implication does not hold in general (see [9, Example 1.1] for a counterexample). If $I$ is a complete intersection in a Cohen-Macaulay ring $R$ with $\operatorname{ara}(I)=: h$, then $\mathrm{H}_{I}^{l}(R) \neq 0 \Longleftrightarrow l=h$ holds. More precisely, at least if $R$ is local, the fact that the case $I$ is a complete intersection can be characterized as follows: Assume that $\mathrm{H}_{I}^{l}(R) \neq 0 \Longleftrightarrow l=h$ holds. Let $r_{1}, \ldots, r_{h} \in I$ be an $R$-regular sequence; then

$$
\sqrt{I}=\sqrt{\left(r_{1}, \ldots, r_{h}\right) R} \Longleftrightarrow r_{1}, \ldots, r_{h} \text { is a regular sequence on } D\left(\mathrm{H}_{I}^{h}(R)\right)
$$

holds (for a proof see [11, Section 0] and for related remarks see [9, Section 3]). Here $D\left({ }_{-}\right):=D_{R}\left({ }_{-}\right)$stands for the Matlis dual functor with respect to the local ring $R$ (i.e. $D\left(\_\right)=\operatorname{Hom}_{R}\left(-, \mathrm{E}_{R}(R / \mathfrak{m})\right)$, where $\mathrm{E}_{R}(M)$ stands for an $R$-injective hull of an $R$-module $M)$ and the phrase that the condition $r_{1}, \ldots, r_{h}$ is a regular sequence on $D\left(\mathrm{H}_{I}^{h}(R)\right.$ means that $D\left(\mathrm{H}_{I}^{h}(R)\right) /\left(r_{1}, \ldots, r_{h}\right) D\left(\mathrm{H}_{I}^{h}(R)\right) \neq 0$ holds and that every $r_{i}$ operates injectively on

$$
D\left(\mathrm{H}_{I}^{h}(R)\right) /\left(r_{1}, \ldots, r_{i-1}\right) D\left(\mathrm{H}_{I}^{h}(R)\right)=D\left(\mathrm{H}_{\left(r_{i}, \ldots, r_{h}\right) R}^{h-i+1}\left(R /\left(r_{1}, \ldots, r_{i-1}\right) R\right)\right) .
$$

Received by the editors April 5, 2006 and, in revised form, January 19, 2007.

2000 Mathematics Subject Classification. Primary 13D45, $13 \mathrm{C} 05$.

Key words and phrases. Local cohomology, Matlis duality, associated prime ideals. 
Thus if one is interested in the arithmetic rank or in complete intersections one is naturally led to consider the set of associated primes of Matlis duals of top local cohomology modules. Similar questions were examined in [11] and [9]. In [11, Section 1] it was conjectured that for every noetherian local ring $(R, \mathfrak{m})$ the equality

$$
\operatorname{Ass}_{R}\left(D\left(\mathrm{H}_{\left(x_{1}, \ldots, x_{i}\right) R}^{i}(R)\right)\right)=\left\{\mathfrak{p} \in \operatorname{Spec}(R) \mid \mathrm{H}_{\left(x_{1}, \ldots, x_{i}\right) R}^{i}(R / \mathfrak{p}) \neq 0\right\}
$$

holds for any $i \geq 1, x_{1}, \ldots, x_{i} \in R$; there it was shown ([11, Theorem 1.1]) that this conjecture $(*)$ is equivalent to: If $(R, \mathfrak{m})$ is a noetherian local ring, $i \geq 1$ and $x_{1}, \ldots, x_{i} \in R$, the set $Y:=\operatorname{Ass}_{R}\left(D\left(\mathrm{H}_{\left(x_{1}, \ldots, x_{i}\right) R}^{i}(R)\right)\right)$ is stable under generalization, i.e.

$$
\mathfrak{p}, \mathfrak{q} \in \operatorname{Spec}(R), \mathfrak{p} \subseteq \mathfrak{q}, \mathfrak{q} \in Y \Rightarrow \mathfrak{p} \in Y
$$

holds. It seems reasonable to conjecture more. Specifically, in the situation above all primes $\mathfrak{p}$ maximal in $Y$ have the same dimension, namely $\operatorname{dim}(R / \mathfrak{p})=i$. We will refer to this conjecture by $(+)$.

In [11, Theorem 2.2.1] it was shown that every noetherian local ring $R$ containing a field

$$
\begin{aligned}
\{\mathfrak{p} \in & \left.\operatorname{Spec}(R) \mid x_{1}, \ldots, x_{i} \text { is part of a system of parameters of } R / \mathfrak{p}\right\} \\
& \subseteq \operatorname{Ass}_{R}\left(D\left(\mathrm{H}_{\left(x_{1}, \ldots, x_{i}\right) R}^{i}(R)\right)\right)
\end{aligned}
$$

holds for any $i \geq 1, x_{1}, \ldots, x_{i} \in R$. Second it was shown (see [11], Corollary 2.2.2) that for every such ring $R$ and every $x \in R$ one has

$$
\operatorname{Ass}_{R}\left(D\left(\mathrm{H}_{x R}^{1}(R)\right)\right)=\operatorname{Spec}(R) \backslash \mathfrak{V}(x) .
$$

Third it is known ([9, Theorem 1.1]) that for an ideal $I$ in a noetherian local ring $R$ such that $0=\mathrm{H}_{I}^{2}(R)=\mathrm{H}_{I}^{3}(R)=\ldots$ holds, one has

$$
\operatorname{ara}(I) \leq 1 \Longleftrightarrow \operatorname{Ass}_{R}\left(D\left(\mathrm{H}_{I}^{1}(R)\right)\right) \text { satisfies prime avoidance }
$$

(see [9, Theorem 1.1 (i) and (ii)] for details).

In section 1 of this work we will generalize the first two of the last three mentioned results to the class of all noetherian local rings; this will be done by methods different from the ones used in [11]; in this section the crucial point is Lemma 1.1. In addition we will prove some more facts related to these results, e.g. a version of the first of the above mentioned results for local cohomology of an $R$-module $M$ (instead of $R$ ).

We would like to point out that Brodmann and Huneke proved Lemma 1.6 (that will be needed in section 2) and used it to give a short proof of the HartshorneLichtenbaum vanishing theorem (see [3]).

In section 2 we consider a $d$-dimensional local ring, an ideal $J$ of $R$ such that $\operatorname{dim}(R / J)=1$ and $\mathrm{H}_{J}^{d}(R)=0$. We are interested in $\operatorname{Ass}_{R}\left(D\left(\mathrm{H}_{J}^{d-1}(R)\right)\right)$. We obtain partial results in the general case; in the case where $R$ is complete we are able to completely compute this set:

$$
\begin{aligned}
\operatorname{Ass}_{R}\left(D\left(\mathrm{H}_{J}^{d-1}(R)\right)\right) & =\{\mathfrak{p} \in \operatorname{Spec}(R) \mid \operatorname{dim}(R / \mathfrak{p})=d-1, \operatorname{dim}(R /(\mathfrak{p}+J))=0\} \\
& \cup \operatorname{Assh} R .
\end{aligned}
$$

Thus $\operatorname{Ass}_{R}\left(D\left(\mathrm{H}_{J}^{d-1}(R)\right)\right.$ is stable under generalization. Assh $(M)$ denotes the set of associated primes of $M$ of highest dimension ( $M$ any $R$-module). 


\section{Preliminaries}

1.1. Lemma. Let $R$ be a ring, and $x, y \in R$ and $U$ an $R$-submodule of $R_{x}$ such that $\operatorname{im} \iota_{x} \subseteq U$, where $\iota_{x}: R \rightarrow R_{x}$ is the canonical map. Let $S:=\operatorname{im} \iota_{y} \subseteq R_{y}$. There exists an $R$-epimorphism

$$
R_{x} / U \rightarrow R_{x y} /\left(S_{x}+U_{y}\right)
$$

Proof. Let $V:=S_{x}+U_{y} \subseteq R_{x y}$ and let $\left(b_{1}, b_{2}, \ldots\right) \in R^{\mathbb{N}^{+}}$be an infinite sequence. For $i \in \mathbb{N}$ we set

$$
\rho_{i}:=\sum_{j=1}^{i} \frac{b_{j}}{x^{i-j+1} y^{j}}+V \in R_{x y} / V \quad(i \in \mathbb{N}) .
$$

We calculate

$$
\begin{aligned}
x \rho_{i+1}-\rho_{i} & =\left(\sum_{j=1}^{i+1} \frac{x b_{j}}{x^{i-j+2} y^{j}}+V\right)-\left(\sum_{j=1}^{i} \frac{b_{j}}{x^{i-j+1} y^{j}}+V\right) \\
& =\frac{b_{i+1}}{y^{i+1}}+V \\
& =0
\end{aligned}
$$

because $\frac{b_{i+1}}{y^{i+1}} \in\left(\operatorname{im} \iota_{x}\right)_{y} \subseteq U_{y} \subseteq V$. Thus we have $x \rho_{i+1}=\rho_{i}$ for all $i \in \mathbb{N}$ and so we get a map $\varphi: R_{x} \rightarrow R_{x y} / V$ given by

$$
\frac{r}{x^{i}} \mapsto r \rho_{i} \quad(r \in R, i \in \mathbb{N}) .
$$

It is easy to see that $\varphi$ is $R$-linear. Let $u \in U$ be arbitrary. There are $r \in R$ and $i \in \mathbb{N}$ such that $u=\frac{r}{x^{i}}$. We have

$$
\varphi(u)=r \rho_{i}=\sum_{j=1}^{i} \frac{r b_{j}}{x^{i-j+1}}+V=u \sum_{j=1}^{i} \frac{x^{j-1} b_{j}}{y^{j}}+V=0,
$$

because $u \sum_{j=1}^{i} \frac{x^{j-1} b_{j}}{y^{j}} \in U_{y} \subseteq V$. This implies $U \subseteq \operatorname{ker}(\varphi)$ and hence we get an induced $R$-homomorphism $f: R_{x} / U \rightarrow R_{x y} / V$. The set $\left\{\frac{1}{x^{i}}+U \mid i \in \mathbb{N}^{+}\right\}$is a generating set for $R_{x} / U$ and so we have

$$
f \text { is surjective } \Longleftrightarrow \varphi \text { is surjective } \Longleftrightarrow\left\{\rho_{1}, \rho_{2}, \ldots\right\} \text { generates } R_{x y} / V \text {. }
$$

The set $\left\{\frac{1}{x^{i} y^{j}}+V \mid i, j \in \mathbb{N}^{+}\right\}$generates $R_{x y} / V$. For $i \in \mathbb{N}^{+}$we set

$$
\mathcal{B}_{i}:=\left(\begin{array}{ccccc}
b_{1} & b_{2} & b_{3} & \ldots & b_{i} \\
b_{2} & b_{3} & b_{4} & \ldots & b_{i+1} \\
\vdots & \vdots & \vdots & & \vdots \\
b_{i} & b_{i+1} & b_{i+2} & \ldots & b_{2 i-1}
\end{array}\right) .
$$

Then we have for $i \in \mathbb{N}^{+}$:

$$
\left(\rho_{i}, y \rho_{i+1}, \ldots, y^{i-1} \rho_{2 i-1}\right)^{T}=\mathcal{B}_{i}\left(\frac{1}{x^{i} y}+V, \frac{1}{x^{i-1} y^{2}}+V, \ldots, \frac{1}{x y^{i}}+V\right)^{T} .
$$

If we choose $b_{1}, b_{2}, \ldots \in R$ in such a way that $\operatorname{det} \mathcal{B}_{i} \in R^{*}$ for all $i \in \mathbb{N}^{+}$(which is possible; $\mathcal{B}$ consists only of ones and zeroes), then $\left\{\rho_{1}, \rho_{2}, \ldots\right\}$ is generating $R_{x y} / V$.

From now on we assume $R$ is noetherian and we can use Cech cohomology. 
1.2. Theorem. Let $R$ be a noetherian ring, $M$ an $R$-module, $m \in \mathbb{N}^{+}, n \in \mathbb{N}$, $x_{1}, \ldots, x_{m}, y_{1}, \ldots, y_{n} \in R$. Then there exists an $R$-epimorphism

$$
\mathrm{H}_{\left(x_{1}, \ldots, x_{m}\right) R}^{m}(M) \rightarrow \mathrm{H}_{\left(x_{1}, \ldots, x_{m}, y_{1}, \ldots, y_{n}\right) R}^{m+n}(M) .
$$

Proof. Obviously it suffices to prove the statement for the case $M=R$. Using Cech cohomology to compute both local cohomology modules the statement follows immediately from Lemma 1.1 by induction on $n$.

1.3. Theorem. Let $(R, \mathfrak{m})$ be a noetherian local ring, $m \in \mathbb{N}^{+}, x_{1}, \ldots, x_{m} \in \mathfrak{m}$ and $M$ a finitely generated $R$-module. Then the following statements hold:

(i) $\operatorname{dim}(M / \mathfrak{p} M) \geq m$ for every $\mathfrak{p} \in \operatorname{Ass}_{R}\left(D\left(\mathrm{H}_{\left(x_{1}, \ldots, x_{m}\right) R}^{m}(M)\right)\right)$.

(ii) $\left\{\mathfrak{p} \in \operatorname{Supp}_{R}(M) \mid x_{1}, \ldots, x_{m}\right.$ is part of a system of parameters of $\left.R / \mathfrak{p}\right\} \subseteq$ $\operatorname{Ass}_{R}\left(D\left(\mathrm{H}_{\left(x_{1}, \ldots, x_{m}\right) R}^{m}(M)\right)\right)$.

(iii) $\operatorname{Ass}_{R}\left(D\left(\mathrm{H}_{x R}^{1}(R)\right)\right)=\operatorname{Spec}(R) \backslash \mathfrak{V}(x)$ for every $x \in R$.

(iv) If $x_{1}, \ldots, x_{m}$ is part of a system of parameters of $M$, we have $\operatorname{Assh}(M) \subseteq$ $\operatorname{Ass}_{R}\left(D\left(\mathrm{H}_{\left(x_{1}, \ldots, x_{m}\right) R}^{m}(M)\right)\right)$; furthermore, in the case $m=\operatorname{dim}(M)$ equality holds: $\operatorname{Ass}_{R}\left(D\left(\mathrm{H}_{\mathfrak{m}}^{\operatorname{dim}(M)}(M)\right)\right)=\operatorname{Assh}(M)$.

(v) If $R$ is complete, $\mathfrak{p} \in \operatorname{Supp}_{R}(M)$ and $\operatorname{dim}(R / \mathfrak{p})=m$, the equivalence $\mathfrak{p} \in \operatorname{Ass}_{R}\left(D\left(\mathrm{H}_{\left(x_{1}, \ldots, x_{m}\right) R}^{m}(M)\right)\right) \Longleftrightarrow x_{1}, \ldots, x_{m}$ is a system of parameters of $R / \mathfrak{p}$ holds.

Proof. We set $I:=\left(x_{1}, \ldots, x_{m}\right) R$.

(i) Let $\mathfrak{p} \in \operatorname{Ass}_{R}\left(D\left(\mathrm{H}_{I}^{m}(M)\right)\right)$. We conclude

$$
0 \neq \operatorname{Hom}_{R}\left(R / \mathfrak{p}, D\left(\mathrm{H}_{I}^{m}(M)\right)\right)=D\left(\mathrm{H}_{I}^{m}(M) \otimes_{R}(R / \mathfrak{p})\right)=D\left(\mathrm{H}_{I}^{m}(M / \mathfrak{p} M)\right) .
$$

Thus we have $\mathrm{H}_{I}^{m}(M / \mathfrak{p} M) \neq 0$, and statement (i) follows.

(ii) Let $\mathfrak{p} \in \operatorname{Supp}_{R}(M)$ such that $x_{1}, \ldots, x_{m}$ is part of a system of parameters of $R / \mathfrak{p}$. By completing $x_{1}, \ldots, x_{m}$ to a system of parameters of $M / \mathfrak{p} M$, and using Theorem 1.2 we may assume that $x_{1}, \ldots, x_{m}$ is a system of parameters of $M / \mathfrak{p} M$. So we have $\operatorname{dim} M / \mathfrak{p} M=\operatorname{dim}(R / \mathfrak{p})=m$. Therefore we get

$$
\begin{aligned}
\operatorname{Hom}_{R}\left(R / \mathfrak{p}, D\left(\mathrm{H}_{I}^{m}(M)\right)\right) & =D\left(\mathrm{H}_{I}^{m}(M / \mathfrak{p} M)\right) \\
& =D\left(\mathrm{H}_{\mathfrak{m}}^{m}(M / \mathfrak{p} M)\right) \\
& \neq 0 .
\end{aligned}
$$

On the other hand we have $\operatorname{Hom}_{R}\left(R / \mathfrak{q}, D\left(\mathrm{H}_{I}^{m}(M)\right)\right)=0$ for every prime ideal $\mathfrak{q}$ of $R$ containing $\mathfrak{p}$ properly by (i); the statement follows.

(iii) Using (ii) it remains to show $x \notin \mathfrak{p}$ for every $\mathfrak{p} \in \operatorname{Ass}_{R}\left(D\left(\mathrm{H}_{x R}^{1}(R)\right)\right)$. As we have seen our hypothesis implies $\mathrm{H}_{x R}^{1}(R / \mathfrak{p}) \neq 0$. So we must have $x \notin \mathfrak{p}$.

(iv) The first statement follows from (ii) and then the second statement from (i).

(v) Let $\mathfrak{p} \in \operatorname{Supp}_{R}(M)$ such that $\operatorname{dim}(R / \mathfrak{p})=m$ and $\mathfrak{p} \in \operatorname{Ass}_{R}\left(D\left(\mathrm{H}_{I}^{m}(M)\right)\right)$. We have to show that $x_{1}, \ldots, x_{m}$ is a system of parameters of $M / \mathfrak{p} M: \mathrm{H}_{I}^{m}(M / \mathfrak{p} M) \neq 0$ implies $\mathrm{H}_{I}^{m}(R / \mathfrak{p}) \neq 0$. As $R$ and hence $R / \mathfrak{p}$ are complete we may conclude from Hartshorne-Lichtenbaum vanishing that $\operatorname{dim}(R /(I+\mathfrak{p}))=0$, i.e. $x_{1}, \ldots, x_{m}$ is a system of parameters of $R / \mathfrak{p}$.

1.4. Remarks. (i) In Theorem 1.3 (ii) we do not have equality in general. For a counterexample see $[11,2.2 .4]$. 
(ii) Statements (ii) and (iii) of Theorem 1.3 are generalizations of $[11$, Theorems 2.2.1 and 2.2.3] resp. of [11, Corollary 2.2.2], where the same statements were shown in special cases depending on the characteristics of $R$ and $R / \mathfrak{m}$.

(iii) Obviously the statements of Theorem 1.3 fit together well with conjectures $(*)$ and $(+)$, but are not sufficient to prove either of them.

1.5. Lemma. Let $(S, \mathfrak{m})$ be a noetherian local complete Gorenstein ring of dimension $n+1(\geq 1)$ and $\mathfrak{P} \subseteq S$ a prime ideal of height $n$. Then

$$
D\left(\mathrm{H}_{\mathfrak{P}}^{n}(S)\right)=\widehat{S_{\mathfrak{P}}} / S
$$

holds canonically.

Proof. This is a special case of [6, Lemma 3.1].

1.6. Lemma. Let $(R, \mathfrak{m})$ be a noetherian local complete domain and $I \subseteq R$ a prime ideal such that $\operatorname{dim}(R / I)=1$. Then there exist a noetherian local complete regular ring $S$, a local homomorphism $S \stackrel{\rho}{\rightarrow} R$ and a prime ideal $\mathfrak{Q} \subseteq R$ such that $R$ is finite as an $S$-module and such that

$$
\operatorname{height}(\operatorname{ker}(\rho))=1, \operatorname{dim}(S / \mathfrak{Q})=1, \sqrt{\mathfrak{Q} R}=I, \operatorname{ker}(\rho) \subseteq \mathfrak{Q}
$$

hold. Furthermore, $R$ and $S$ have the same residue field via $\rho$.

Proof. See [3, Proof of (1.2)].

\section{Results}

In this section we calculate the set of associated primes of $D\left(\mathrm{H}_{J}^{d-1}(R)\right)$, where $J$ is a one-dimensional ideal in a $d$-dimensional local complete ring $R$; furthermore we will partially calculate this set in the more general situation where $R$ is not necessarily complete.

2.1. Lemma. Let $R$ be a noetherian ring.

(i) Let $\mathfrak{P}$ be a prime ideal of $R$ which is not maximal. Then the equivalence

$$
R_{\mathfrak{P}}=\widehat{R_{\mathfrak{P}}} \Longleftrightarrow \mathfrak{P} \text { is minimal in } \operatorname{Spec}(R)
$$

holds.

(ii) Assume that $R$ is local (and noetherian) and that all prime ideals associated to $R$ are minimal in $\operatorname{Spec}(R)$. Then $\operatorname{Ass}_{R}(\hat{R} / R) \subseteq \operatorname{Ass}(R)$ holds. In particular if $R$ is a non-complete (local) domain (i.e. if $R \subsetneq \hat{R}$ ),

$$
\operatorname{Ass}_{R}(\hat{R} / R)=\{0\}
$$

holds.

Proof. (i) The implication $\Leftarrow$ is clear as every zero-dimensional local noetherian ring is complete. We assume there exists a prime ideal $P$ of $R$ which is neither minimal nor maximal in $\operatorname{Spec}(R)$ and such that $R_{P}=\widehat{R_{P}} . P R_{P}$ is not minimal in $\operatorname{Spec}(R)$. Choose $Q, Q^{\prime} \in \operatorname{Spec}(R)$ such that $Q^{\prime} \subsetneq P \subsetneq Q$ and such that $\operatorname{dim}\left(R_{Q} / P R_{Q}\right)=1$. We set $\mathfrak{R}:=R_{Q} / Q^{\prime} R_{Q}$ and $\mathfrak{P}:=P R_{Q} / Q^{\prime} R_{Q} \in \operatorname{Spec}(\mathfrak{R})$ and we get

$$
\mathfrak{R}_{P}=R_{P} / Q^{\prime} R_{P}=\widehat{R_{P}} / Q^{\prime} \widehat{R_{P}}=\widehat{R_{P} / Q^{\prime} R_{P}}=\widehat{\mathfrak{R}_{P}} .
$$

So we may assume that $R$ is a local domain and $\operatorname{dim}(R / P)=1$.

Take $y \in \mathfrak{m} \backslash P$. Assume that for some $n \in \mathbb{N}$

$$
P^{(n)} \subseteq P^{(n+1)}+y R
$$


holds $\left(P^{(n)}:=P^{n} R_{P} \cap R\right.$ is a $P$-primary ideal of $R$ such that $\left.P^{(n)} R_{P}=P^{n} R_{P}\right)$. It would follow that

$$
P^{(n)}=P^{(n)} \cap\left(P^{(n+1)}+y R\right)=P^{(n+1)}+\left(P^{(n)} \cap y R\right)=P^{(n+1)}+y P^{(n)}
$$

and then $P^{(n)}=P^{(n+1)}$ by the Nakayama lemma. Again by Nakayama, this would imply $P^{n} R_{P}=0$, and so $P R_{P}$ would be minimal in $\operatorname{Spec}\left(R_{P}\right)$. We conclude that for every $n \in \mathbb{N}$

$$
P^{(n)} \nsubseteq P^{(n+1)}+y R
$$

holds. For every $n \in \mathbb{N}$ we choose $x_{n} \in P^{(n)} \backslash\left(P^{(n+1)}+y R\right)$ and define (for every $n \in \mathbb{N}^{+}$)

$$
\xi_{n}:=\sum_{i=0}^{n-1} \frac{x_{i}}{y^{(i+1)^{2}}} \in R_{P} .
$$

Because of $\xi_{n+1}-\xi_{n}=\frac{x_{n}}{y^{(n+1)^{2}}} \in P^{n} R_{P}$ (for every $n$ ), we have

$$
\left(\xi_{n}+P^{n} R_{P}\right)_{n \in \mathbb{N}^{+}} \in \widehat{R_{P}}=R_{P} .
$$

There exists a $\xi \in R_{P}$ such that

$$
\left(\xi+P^{n} R_{P}\right)_{n \in \mathbb{N}^{+}}=\left(\xi_{n}+P^{n} R_{P}\right)_{n \in \mathbb{N}^{+}},
$$

i.e.

$$
\xi-\xi_{n} \in P^{n} R_{P}
$$

for all $n \in \mathbb{N}^{+}$.

Write $\xi=\frac{a}{b}$, where $a \in R, b \in R \backslash P$. The ideal $P+b R$ of $R$ is either $R$ or $\mathfrak{m}$-primary, so there exist $p \in \mathbb{N}^{+}$and $c \in R$ such that $y^{p}-b c \in P$. It follows that

$$
y^{p n}-b c_{n} \in P^{n},
$$

where

$$
c_{n}:=b^{-1}\left(y^{p n}-\left(y^{p}-b c\right)^{n}\right) \in R
$$

and we conclude that

$$
\xi-\frac{a c_{n}}{y^{p n}}=\frac{a y^{p n}-a b c_{n}}{b y^{p n}}=\frac{a\left(y^{p}-b c\right)^{n}}{b y^{p n}} \in P^{n} R_{P}
$$

for every $n \in \mathbb{N}^{+}$. We get

$$
\xi_{n}-\frac{a c_{n}}{y^{p n}}=\xi-\frac{a c_{n}}{y^{p n}}-\left(\xi-\xi_{n}\right) \in P^{n} R_{P}
$$

for every $n \in \mathbb{N}^{+}$. From this we get (for $n>p$ ) after multiplication by $y^{n^{2}}$ that

$$
\sum_{i=0}^{n-1} x_{i} y^{n^{2}-(i+1)^{2}}-a c_{n} y^{n(n-p)} \in P^{(n)}
$$

and in particular $x_{n-1} \in P^{(n)}+y R$, which is a contradiction.

(ii) We have to prove only the first statement; the second one follows from it immediately. Let $P$ be an arbitrary element of $\operatorname{Spec}(R) \backslash \operatorname{Ass}(R)$. We conclude $\operatorname{Hom}_{R}(R / P, R)=0$ and hence also $\operatorname{Hom}_{R}(R / P, \hat{R})=0$ (because $P$ contains an element which operates injectively on $R$ and $\hat{R}$ is flat over $R$ ). Thus the short exact sequence

$$
0 \rightarrow R \stackrel{\subseteq}{\rightrightarrows} \hat{R} \rightarrow \hat{R} / R \rightarrow 0
$$


induces an exact sequence

$$
0 \rightarrow \operatorname{Hom}_{R}(R / P, \hat{R} / R) \rightarrow \operatorname{Ext}_{R}^{1}(R / P, R) \stackrel{\varphi}{\rightarrow} \operatorname{Ext}_{R}^{1}(R / P, \hat{R}) .
$$

By our hypothesis there exists $x \in P$ such that $x \notin Q$ for all $Q \in \operatorname{Ass}(R)$. We get short exact sequences

$$
0 \rightarrow R \stackrel{x}{\rightarrow} R \rightarrow R / x R \rightarrow 0
$$

and

$$
0 \rightarrow \hat{R} \stackrel{x}{\rightarrow} \hat{R} \rightarrow \hat{R} / x \hat{R} \rightarrow 0 .
$$

Because of $x \in P$ a commutative diagram with exact rows is induced:

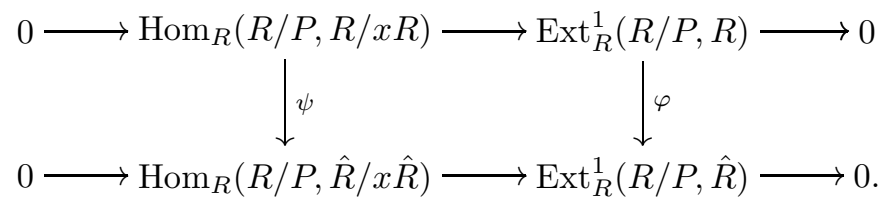

$\psi$ is injective as $R / x R \subseteq \widehat{R / x R}=\hat{R} / x \hat{R}$. Therefore $\varphi$ is injective, which implies that $\operatorname{Hom}_{R}(R / P, \hat{R} / R)=0$, i.e. $P \notin \operatorname{Ass}_{R}(\hat{R} / R)$.

2.2. Theorem. Let $(R, \mathfrak{m})$ be a d-dimensional local noetherian complete domain, where $d \geq 1$; let $P$ be a prime ideal of $R$ such that $\operatorname{dim}(R / P)=1$. Then

$$
\{0\} \in \operatorname{Ass}_{R}\left(D\left(\mathrm{H}_{P}^{d-1}(R)\right)\right)
$$

holds.

Proof. We apply Lemma 1.6, set $R_{0}:=\operatorname{im}(\rho)$ and consider the ideal $\mathfrak{Q}$ from Lemma 1.6 as an ideal of $R_{0}$. By Lemma 1.6, $R_{0}$ is a complete intersection, in particular it is Gorenstein. By $\mathfrak{m}_{0}$ we denote the maximal ideal of $R_{0} . R$ is finite over $R_{0}$ and so we have $D_{R_{0}}(R)=\operatorname{Hom}_{R_{0}}\left(R, \mathrm{E}_{R_{0}}\left(R_{0} / \mathfrak{m}_{0}\right)\right)=\mathrm{E}_{R}(R / \mathfrak{m})=D_{R}(R)$, which implies $D_{R_{0}}(M)=D_{R}(M)$ for every $R$-module $M$. On the other hand the functor $\mathrm{H}_{\mathfrak{Q}}^{d-1}\left(\_\right)$ is right exact by Hartshorne-Lichtenbaum vanishing; in particular we have

$$
D_{R}\left(\mathrm{H}_{\mathfrak{P}}^{d-1}(R)\right)=D_{R_{0}}\left(\mathrm{H}_{\mathfrak{Q}}^{d-1}\left(R_{0}\right) \otimes_{R_{0}} R\right)=\operatorname{Hom}_{R_{0}}\left(R, D_{R_{0}}\left(\mathrm{H}_{\mathfrak{Q}}^{d-1}\left(R_{0}\right)\right)\right),
$$

and so every $R_{0}$-monomorphism $R_{0} \rightarrow D_{R_{0}}\left(\mathrm{H}_{\mathfrak{Q}}^{d-1}\left(R_{0}\right)\right)$ induces an $R$-monomorphism $\operatorname{Hom}_{R_{0}}\left(R, R_{0}\right) \rightarrow D_{R}\left(\mathrm{H}_{\mathfrak{P}}^{d-1}(R)\right)$. But $\{0\} \in \operatorname{Ass}_{R_{0}}\left(\operatorname{Hom}_{R_{0}}\left(R, R_{0}\right)\right)$ holds (because

$$
\operatorname{Hom}_{R_{0}}\left(R, R_{0}\right) \otimes_{R_{0}} Q\left(R_{0}\right)=\operatorname{Hom}_{Q\left(R_{0}\right)}\left(R \otimes_{R_{0}} Q\left(R_{0}\right), Q\left(R_{0}\right)\right)
$$

is a non-zero $Q\left(R_{0}\right)$-vector space; here we use the fact that $R$ is finite over $\left.R_{0}\right)$, and thus it suffices to show $\{0\} \in \operatorname{Ass}_{R_{0}}\left(D_{R_{0}}\left(\mathrm{H}_{\mathfrak{Q}}^{d-1}\left(R_{0}\right)\right)\right)$, i.e. we may assume $R_{0}$ is Gorenstein. Now, by Lemma 1.5, we have a commutative diagram with exact rows:

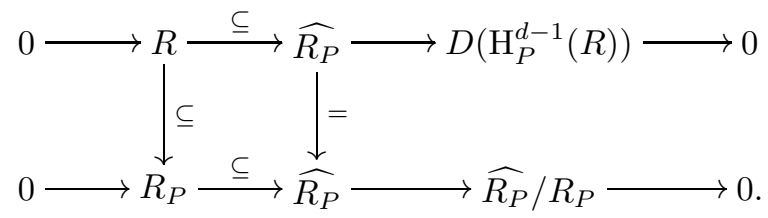

This diagram induces an epimorphism

$$
D\left(\mathrm{H}_{P}^{d-1}(R)\right) \rightarrow \widehat{R_{P}} / R_{P}
$$


By Lemma 2.1 (i) we have $\widehat{R_{P}} / R_{P} \neq 0$, and it follows from Lemma 2.1 (ii) that $\left(\widehat{R_{P}} / R_{P}\right) \otimes_{R}(Q(R)) \neq 0$. Thus we have $D\left(\mathrm{H}_{P}^{d-1}(R)\right) \otimes_{R} Q(R) \neq 0$ by the above epimorphism.

2.3. Lemma. Let $(R, \mathfrak{m})$ be a noetherian local complete ring, $d:=\operatorname{dim}(R) \geq 1$, and $J \subseteq R$ an ideal of $R$ such that $\operatorname{dim}(R / J)=1$. Then

$\left\{Q \in \operatorname{Ass}_{R}\left(D\left(\mathrm{H}_{J}^{d-1}(R)\right)\right) \mid \operatorname{dim}(R / Q)=d\right\}=\{Q \in \operatorname{Assh}(R) \mid \operatorname{dim}(R /(J+Q)) \geq 1\}$. If $\mathrm{H}_{J}^{d}(R)=0$,

$$
\operatorname{Assh}\left(D\left(\mathrm{H}_{J}^{d-1}(R)\right)\right)=\operatorname{Assh}(R) .
$$

Proof. The second statement follows from the first one by Hartshorne-Lichtenbaum vanishing. We now prove the first statement in the special case where

$$
\operatorname{dim}(R /(J+Q)) \geq 1
$$

for all $Q \in \operatorname{Assh}(R)$. In the second part of the proof we will show how to reduce the general to the special situation. Now, in the special case it suffices to show the inclusion " $\supseteq$ ". By Hartshorne-Lichtenbaum vanishing we have $\mathrm{H}_{J}^{d}(R)=0$, i.e. $\mathrm{H}_{J}^{d-1}($ ( ) is right exact. Let $Q \in \operatorname{Assh}(R)$ be arbitrary. The canonical epimorphism $R \rightarrow R / Q=: \bar{R}$ induces a monomorphism

$$
D_{\bar{R}}\left(\mathrm{H}_{J \bar{R}}^{d-1}(\bar{R})\right)=D_{R}\left(\mathrm{H}_{J}^{d-1}(\bar{R})\right) \rightarrow D\left(\mathrm{H}_{J}^{d-1}(R)\right) .
$$

If $\{0\} \in \operatorname{Ass}_{\bar{R}}\left(D_{\bar{R}}\left(\mathrm{H}_{J \bar{R}}^{d-1}(\bar{R})\right)\right)$, then $Q \in \operatorname{Ass}_{R}\left(D\left(\mathrm{H}_{J}^{d-1}(R)\right)\right)$, and so we may assume that $R$ is a domain. If we can write $J=J_{1} \cap J_{2}$ with ideals $J_{1}, J_{2}$ of $R$ such that $J_{1}+$ $J_{2}$ is $\mathfrak{m}$-primary, then because of $\mathrm{H}_{J_{1}}^{d}(R)=\mathrm{H}_{J_{2}}^{d}(R)=0$ (Hartshorne-Lichtenbaum vanishing) a Mayer-Vietoris sequence argument gives us an epimorphism

$$
\mathrm{H}_{J}^{d-1}(R) \rightarrow \mathrm{H}_{J_{1}+J_{2}}^{d}(R)=\mathrm{H}_{\mathfrak{m}}^{d}(R) .
$$

But then Theorem 1.3 shows that

$$
\{0\}=\operatorname{Assh}(R)=\operatorname{Ass}_{R}\left(D\left(\mathrm{H}_{\mathfrak{m}}^{d}(R)\right)\right) \subseteq \operatorname{Ass}_{R}\left(D\left(\mathrm{H}_{J}^{d-1}(R)\right)\right) .
$$

If there is no such decomposition $J=J_{1} \cap J_{2}$ of $J$ we may assume that $J$ is a prime ideal; but then the statement follows from Theorem 2.2. Now we turn to the general case, i.e. we assume there is a $Q \in \operatorname{Assh}(R) \operatorname{such}$ that $\operatorname{dim}(R /(J+Q))=0$. We define $U(R)$ to be the intersection of all $Q^{\prime}$-primary components of a primary decomposition of the zero ideal in $R$ for all $Q^{\prime} \in \operatorname{Assh}(R)$. Apparently we have $\operatorname{Ass}_{R}(R / U(R))=\operatorname{Assh}(R)$ and $\operatorname{dim}(U(R))<d$. Because of the latter fact the short exact sequence $0 \rightarrow U(R) \stackrel{\subseteq}{\rightarrow} R \rightarrow R / U(R) \rightarrow 0$ induces an exact sequence

$$
0 \rightarrow D\left(\mathrm{H}_{J}^{d-1}(R / U(R))\right) \rightarrow D\left(\mathrm{H}_{J}^{d-1}(R)\right) \rightarrow D\left(\mathrm{H}_{J}^{d-1}(U(R))\right) .
$$

Trivially $\operatorname{dim}_{R}\left(\operatorname{Supp}_{R}\left(\mathrm{H}_{J}^{d-1}(U(R))\right)\right) \leq d-1$ holds. By considering $R / U(R)$ rather than $R$ we may assume that $\operatorname{Ass}_{R}(R)=\operatorname{Assh}(R)$. We write $0=I^{\prime} \cap I^{\prime \prime}$ with ideals $I^{\prime}, I^{\prime \prime}$ of $R$ such that $\operatorname{Ass}_{R}(R)=\operatorname{Ass}_{R}\left(R / I^{\prime}\right) \cup \operatorname{Ass}_{R}\left(R / I^{\prime \prime}\right)$ and $\operatorname{dim}\left(R /\left(J+Q^{\prime}\right)\right) \geq 1$ for all $Q^{\prime} \in \operatorname{Ass}_{R}\left(R / I^{\prime}\right)$ and $\operatorname{dim}\left(R /\left(J+Q^{\prime \prime}\right)\right)=0$ for all $\left.Q^{\prime \prime} \in \operatorname{Ass}_{R}\left(R / I^{\prime \prime}\right)\right)$. It follows that $\operatorname{dim}\left(R /\left(J+I^{\prime \prime}\right)\right)=0$. By using a Mayer-Vietoris argument and the facts that $\mathrm{H}_{J}^{d}\left(R / I^{\prime}\right)=0$ (Hartshorne-Lichtenbaum vanishing) and $\mathrm{H}_{J}^{i}\left(R / I^{\prime \prime}\right)=$ $\mathrm{H}_{\mathfrak{m}}^{i}\left(R / I^{\prime \prime}\right)$ for all $i \in \mathbb{N}$, we get a short exact sequence

$$
\begin{gathered}
D\left(\mathrm{H}_{\mathfrak{m}}^{d-1}\left(R / I^{\prime}+I^{\prime \prime}\right)\right) \rightarrow D\left(\mathrm{H}_{J}^{d-1}\left(R / I^{\prime}\right)\right) \oplus D\left(\mathrm{H}_{\mathfrak{m}}^{d-1}\left(R / I^{\prime \prime}\right)\right) \\
\rightarrow D\left(\mathrm{H}_{J}^{d-1}(R)\right) \rightarrow D\left(\mathrm{H}_{\mathfrak{m}}^{d-2}\left(R /\left(I^{\prime}+I^{\prime \prime}\right)\right)\right) .
\end{gathered}
$$


It is clear that we have

$\operatorname{dim}_{R}\left(\operatorname{Supp}_{R}\left(D\left(\mathrm{H}_{\mathfrak{m}}^{d-1}\left(R /\left(I^{\prime}+I^{\prime \prime}\right)\right)\right)\right)\right), \operatorname{dim}_{R}\left(\operatorname{Supp}_{R}\left(D\left(\mathrm{H}_{\mathfrak{m}}^{d-2}\left(R /\left(I^{\prime}+I^{\prime \prime}\right)\right)\right)\right)\right) \leq d-1$.

One can write $R$ as a quotient of a local Gorenstein $\operatorname{ring} S$ such that $\operatorname{dim}(S)=$ $\operatorname{dim}(R)$; over $S$ one has local duality and therefore one may conclude (note that in the formula below it does not make any difference if we take $D$ with respect to $R$ or to $S)$

$$
D\left(\mathrm{H}_{\mathfrak{m}}^{d-1}\left(R / I^{\prime \prime}\right)\right)=\operatorname{Ext}_{S}^{1}\left(R / I^{\prime \prime}, S\right)
$$

and hence

$$
\operatorname{dim}_{R}\left(\operatorname{Supp}_{R}\left(D\left(\mathrm{H}_{\mathfrak{m}}^{d-1}\left(R / I^{\prime \prime}\right)\right)\right)\right) \leq d-1 .
$$

Thus we get, by what has already been shown,

$$
\begin{aligned}
& \left\{Q \in \operatorname{Ass}_{R}\left(D\left(\mathrm{H}_{J}^{d-1}(R)\right)\right) \mid \operatorname{dim}(R / Q)=d\right\} \\
& \quad=\left\{Q \in \operatorname{Ass}_{R}\left(D\left(\mathrm{H}_{J}^{d-1}\left(R / I^{\prime}\right)\right)\right) \mid \operatorname{dim}(R / Q)=d\right\}=\operatorname{Assh}\left(R / I^{\prime}\right) .
\end{aligned}
$$

2.4. Theorem. Let $(R, \mathfrak{m})$ be a d-dimensional noetherian local ring and $J \subseteq R$ an ideal such that $\operatorname{dim}(R / J)=1$ and $\mathrm{H}_{J}^{d}(R)=0$. Then

$$
\operatorname{Assh}(R)=\operatorname{Assh}\left(D\left(\mathrm{H}_{J}^{d-1}(R)\right)\right) \text {. }
$$

Proof. One has $\mathrm{H}_{J \hat{R}}^{d}(\hat{R})=\mathrm{H}_{J}^{d}(R) \otimes_{R} \hat{R}=0$ and

$$
\begin{aligned}
D_{\hat{R}}\left(\mathrm{H}_{J \hat{R}}^{d-1}(\hat{R})\right) & =D_{\hat{R}}\left(\mathrm{H}_{J}^{d-1}(R) \otimes \hat{R}\right) \\
& =\operatorname{Hom}_{R}\left(\mathrm{H}_{J}^{d-1}(R), D_{\hat{R}}(\hat{R})\right) \\
& =D_{R}\left(\mathrm{H}_{J}^{d-1}(R)\right) .
\end{aligned}
$$

These imply that every $\hat{R}$-monomorphism $\varphi: \hat{R} / \mathfrak{P} \rightarrow D_{\hat{R}}\left(\mathrm{H}_{J \hat{R}}^{d-1}(\hat{R})\right)$, where $\mathfrak{P}$ is a prime ideal of $\hat{R}$, induces an $R$-monomorphism $\hat{R} / \mathfrak{P} \rightarrow D_{R}\left(\mathrm{H}_{J}^{d-1}(R)\right)$. On the other hand we have an $R$-monomorphism $R / \mathfrak{p} \rightarrow \hat{R} / \mathfrak{P}$, where $\mathfrak{p}:=\mathfrak{P} \cap R$. Composition of these monomorphisms gives us a monomorphism

$$
R / \mathfrak{p} \rightarrow D_{R}\left(\mathrm{H}_{J}^{d-1}(R)\right) .
$$

Because of $\operatorname{Assh}(R)=\{\mathfrak{P} \cap R \mid \mathfrak{P} \in \operatorname{Assh}(\hat{R})\}$ we may assume $R$ is complete. But then the statement follows from Lemma 2.3.

2.5. Theorem. Let $R$ be a d-dimensional local complete ring and $J \subseteq R$ an ideal such that $\operatorname{dim}(R / J)=1$ and $\mathrm{H}_{J}^{d}(R)=0$. Then

$$
\begin{aligned}
\operatorname{Ass}_{R}\left(D\left(\mathrm{H}_{J}^{d-1}(R)\right)\right) & =\{P \in \operatorname{Spec}(R) \mid \operatorname{dim}(R / P)=d-1, \operatorname{dim}(R /(P+J))=0\} \\
& \cup \operatorname{Assh}(R) .
\end{aligned}
$$

Proof. Let $P \in \operatorname{Spec}(R)$. If $\operatorname{dim}(R / P) \leq d-2$ we have $\operatorname{Hom}_{R}\left(R / P, D\left(\mathrm{H}_{J}^{d-1}(R)\right)\right)=$ $D\left(\mathrm{H}_{J}^{d-1}(R / P)\right)=0$ and hence $P \notin \operatorname{Ass}_{R}\left(D\left(\mathrm{H}_{J}^{d-1}(R)\right)\right)$. If $\operatorname{dim}(R / P)=1$, then $(\bar{R}:=R / P)$ :

$$
\operatorname{Hom}_{R}\left(R / P, D\left(\mathrm{H}_{J}^{d-1}(R)\right)\right)=D\left(\mathrm{H}_{J}^{d-1}(R / P)\right)=D\left(\mathrm{H}_{J \bar{R}}^{d-1}(\bar{R})\right) .
$$

$R$ is complete and so, by Hartshorne-Lichtenbaum, the equivalence

$$
\mathrm{H}_{J \bar{R}}^{d-1}(\bar{R}) \neq 0 \Longleftrightarrow \operatorname{dim}(\bar{R} / J \bar{R})=0
$$


holds. On the other hand we have $\bar{R} / J \bar{R}=R /(P+J)$ and therefore we have

$$
\begin{gathered}
\left\{P \in \operatorname{Ass}_{R}\left(D\left(\mathrm{H}_{J}^{d-1}(R)\right)\right) \mid \operatorname{dim}(R / P)=d-1\right\} \\
=\{P \in \operatorname{Spec}(R) \mid \operatorname{dim}(R / P)=d-1, \operatorname{dim}(R /(P+J))=0 .
\end{gathered}
$$

Now the statement follows from Lemma 2.3.

\section{REFERENCES}

[1] Bass, H. On the ubiquity of Gorenstein rings, Math. Z. 82 (1963) 8-28. MR0153708 (27:3669)

[2] Brodmann, M. and Hellus, M. Cohomological patterns of coherent sheaves over projective schemes, Journal of Pure and Applied Algebra 172 (2002) 165-182. MR1906872 (2003f:13017)

[3] Brodmann, M. and Huneke, C. A quick proof of the Hartshorne-Lichtenbaum vanishing theorem, Algebraic geometry and its applications, Springer, New York (1994) 305-308. MR1272037 (95b:13022)

[4] Brodmann, M. and Sharp, R. J. Local Cohomology, Cambridge Studies in Advanced Mathematics 60 (1998). MR1613627 (99h:13020)

[5] Bruns, W. and Herzog, J. Cohen-Macaulay Rings, Cambridge University Press (1993). MR1251956 (95h:13020)

[6] Chiriacescu, G. Cofiniteness of local cohomology modules over regular local rings, Math. Soc. 32 (2000) 1-7. MR1718769 (2000k:13014)

[7] Eisenbud, D. Commutative Algebra with A View Toward Algebraic Geometry, Springer Verlag (1995). MR1322960 (97a:13001)

[8] Grothendieck, A. Local Cohomology, Lecture Notes in Mathematics, Springer Verlag (1967). MR0224620 (37:219)

[9] Hellus, M. Matlis duals of top local cohomology modules and the arithmetic rank of an ideal, Comm. Algebra 35, no. 4 (2007), 1421-1432.

[10] Hellus, M. On the set of associated primes of a local cohomology module, J. Algebra 237 (2001) 406-419. MR1813886 (2001m:13023)

[11] Hellus, M. On the associated primes of Matlis duals of top local cohomology modules, to appear in Communications in Algebra 33. MR2183976 (2006h:13026)

[12] Huneke, C. Problems on Local Cohomology, Res. Notes Math. 2 (1992) 93-108. MR1165320 (93f:13010)

[13] Huneke, C. and Lyubeznik, G. On the vanishing of local cohomology modules, Invent. Math. 102 (1990) 73-93. MR1069240 (91i:13020)

[14] Matlis, E. Injective modules over Noetherian rings, Pacific J. Math. 8 (1958) 511-528. MR0099360 (20:5800)

[15] Matsumura, H. Commutative ring theory, Cambridge University Press (1986). MR879273 (88h:13001)

[16] Scheja, G. and Storch, U. Regular Sequences and Resultants, AK Peters (2001). MR1831871 (2002b:13011)

Department of Mathematics, University of Leipzig, D-04109 Leipzig, Germany

E-mail address: michael.hellus@math.uni-leipzig.de

Department of Mathematics, University of Leipzig, D-04109 Leipzig, Germany

E-mail address: juergen.stueckrad@math.uni-leipzig.de 